\title{
CONNECTING CLIMATE INFORMATION WITH HEALTH OUTCOMES
}

\author{
Madeleine C. Thomson, C. Jessica E. Metcalf \\ and Simon J. Mason \\ Contributors: Adrian M. Tompkins and Mary Hayden
}

Happy the man who has been able to learn the causes of things

Georgics, II by Virgil c. 29BC

\subsection{Introduction}

In agricultural development water is understood as a precious and finite resource that must be used wisely to maximize crop growth. The relationship between available water and crop growth is dependent on soil type, plant cultivar and development stage, and is relatively easy to calculate; after all, the plant stays in the same place throughout its growing period. In contrast, pathogens and animal and human hosts exhibit a complex set of biological and behavioural interactions in response to climatic and environmental drivers that may vary in both time and space. As climate may be a significant driver of a wide range of health outcomes (see Chapter 2), climate information can potentially support a wide range of health decisions (see Box 3.1).

All decision processes involved in the prevention or control of climate-sensitive health issues (such as the decision to spray houses with indoor residual insecticides for the control of malaria-bearing mosquitoes) have their own spatial or temporal context. Understanding this context, including climatic, environmental and population characteristics, is the first step to using climate information effectively. The spatial or temporal dynamics of the problem as well as potential solution(s) are key issues in decision-making processes. For instance, heat waves may be of particular concern to elderly populations in urban environments. Prevention/control decisions for heat waves may be made at the individual level, the local administrative level or may require a regional or national process. They may be routine control efforts enacted prior to the seasonal occurrence of heat waves. Alternatively, control 


\section{BOX 3.1. CLIMATE INFORMATION OPPORTUNITIES}

Climate information has the potential to inform a wide range of health decisions ${ }^{1}$ through an improved understanding of the following:

- Mechanisms of Disease Transmission: to help identify new opportunities for intervention.

- Spatial Risk: to help identify populations at risk for better targeting of interventions.

- Seasonal Risk: to inform the timing of routine interventions.

- Sub-seasonal and Year-to-Year Changes in Risk: to identify when changes in epidemic risk are likely to occur to initiate appropriate prevention and response strategies.

- Trends in Risk: to identify long-term drivers of disease occurrence (including shifts in the climate) to plan for and support future prevention and response strategies.

- Assessment of the Impacts of Interventions: to remove the role of climate if it interferes with the proper assessment of interventions.

efforts may be initiated in response to occasional extreme events that visit a region during an unusual year. Changes in control strategies (including urban planning) may also be undertaken in response to observed shifts in the underlying frequency and intensity of heat waves.

In this chapter we consider a range of factors that need to be taken into account when seeking to use climate information to improve health decision-making. Identifying causal mechanisms that link climate drivers with specific health issues is an important starting point for policy-makers. Matching decision time-horizons to climate information in a way that takes account of scale issues, uncertainties in the underlying data and modelling approaches as well as institutional barriers to knowledge and data sharing is also critical. And of course, all of this is dependent on a solid understanding of the climate information (including its limitations) that is available to health decision-makers. A researcher may be satisfied with a simple times-series of climate data from an authoritative source; a decision-maker needs to know that the climate information is robust, available for routine use and scalable (i.e., can be used over the entire region of interest).

\subsection{Climate information for use in health decision-making}

Climate is measured routinely around the world by the meteorological/climate community using internationally agreed standards, with a significant amount of data shared freely in real-time $(\mathbb{S} 6.2)$. Historical and current climate data and 
information products ( $\int S 6.3$ and 6.4) can be used to inform a wide range of planning processes as well as in early warning systems (EWS). Information about the future weather and/or climate varies in specificity (lead-time, spatial and temporal averaging), the regions and seasons where it is most accurate and its status in terms of operational delivery. Information that is currently available across the globe for routine operational decision-making can be divided into three specific timeframes: weather, season and climate change. These timescales are related to spatial scale: at the one extreme, short-term weather predictions are reliable at the local level, while, at the other, climate change trends (especially temperature) are most reliable at the subcontinental level. Climate change scenarios, indicating possible long-term changes in temperature and rainfall, provide important guidance to climate change mitigation, motivating reductions in carbon emissions from the health sector itself and promoting the health co-benefits of a low-carbon economy. When it comes to adapting to climate variability and change, information about possible future climates is important for planning major infrastructure developments or considering long-term policy shifts (e.g., from malaria control to malaria eradication ${ }^{2}$ ). However, most health programming decisions are made at seasonal to annual timescales or respond to four- to five-year political or funding cycles. ${ }^{3}$ Timescales of climate information need to be matched with these time horizons of decision-making (Table 3.1).

Weather can be predicted at the local scale for several days ahead with a reasonable level of accuracy (particularly in the extratropics), but the accuracy after three to five days has deteriorated considerably (see Chapter 7). The seasonal climate

TABLE 3.1 Time horizons for decision-making in the health sector

Investment

$2-5$ decades

Strategic planning

6-20 years

Policy cycles

2-5 years

Planning cycles

$<2$ yrs

Seasonal preparedness and response

$<4$ months

Weekly facility management

$<1$ week
Carbon emissions mitigation strategies

Malaria eradication strategies

Major infrastructure investment

Workforce development

Research and development of medical countermeasures (e.g., drugs, vaccines) and vector control tools (e.g., new insecticides) Improved nutritional content of crops Health facility investments Curriculum development

4- to 5-year political cycle

Health service re-organization

2- to 5-year research grant cycle

Annual planning and commissioning cycle Demand for visible 'quick wins' from funders Seasonal planning cycle Epidemic/disaster preparedness and response Weather disaster preparedness and response Patient scheduling for non-urgent cases 


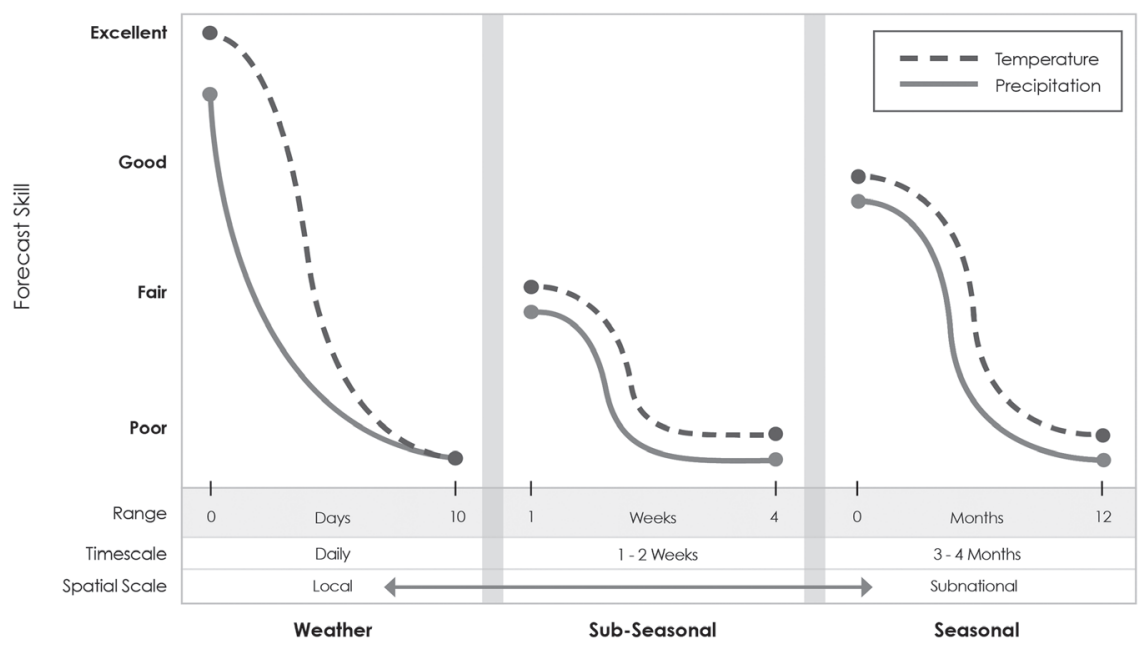

FIGURE 3.1 Best forecast skill at multiple timescales with indications of the forecast ranges, timescales and spatial scales over which the forecasts are averaged

may be affected by phenomena such as the El Niño - Southern Oscillation (ENSO; see Box 5.1), giving advanced warning of unusual rainfall or temperature months in advance. The ENSO and other influences underpin seasonal climate forecasts, which are most robust in the tropics (see Chapter 8). Projections of trends in 30-year averaged climate due to greenhouse gas emissions are available for the long-term and are considered robust for temperature (see Chapter 9). Three other timeframes are only briefly considered in this book because they remain, for now, substantially in the research arena, namely sub-seasonal, multi-annual and decadal. Emerging capacities in forecast capability at sub-seasonal timescales (e.g., seven-day averaged weather two weeks or more in advance) and multi-annual timescales provide new opportunities for health research, but evidence to date of potential utility is for specific locations only. Long sought for decadal prediction (five- to ten-year averaged climate going out over ten- to 30-year timescales) is a focus of intense research. A schematic representation of predictability of anomalies at the shorter verifiable timescales is presented in Figure 3.1.

\subsection{Data issues}

In order to facilitate climate and health analyses, data must be shared between communities. Data sharing is an issue in nearly every organization because there are consequences, both good and bad, to sharing information beyond institutional borders. There are many barriers to sharing health data, even within the health community (see Table 3.2), ${ }^{4}$ and these barriers can be even greater when it comes to sharing data between different sectors, such as climate and health. There are also significant barriers to the sharing of climate data, especially observations from meteorological stations at high temporal resolution - e.g., daily data (see $\$ S$ 6.4.1 and Box 6.1). 
TABLE 3.2 Evidence for barriers to sharing of routinely collected public health data ${ }^{4}$

\section{Technical}

1. Data not collected

2. Data not preserved

3. Data not found

4. Language barrier

5. Restrictive data format

6. Technical solutions not available

7. Lack of metadata and standards

\section{Motivational}

8. No incentives

9. Opportunity cost

10. Possible criticism

11. Disagreement on data use

\section{Economic}

12. Possible economic damage

13. Lack of resources

\section{Political}

14. Lack of trust

15. Restrictive policies

16. Lack of guidelines

\section{Legal}

17. Ownership and copyright

18. Protection of privacy

\section{Ethical}

19. Lack of proportionality

20. Lack of reciprocity

Increasingly, countries are developing Open Data policies, where government information is made visible and available. The main goal is to harness the data revolution for sustainable development ${ }^{5}$ with a focus on climate, health and agriculture. Open data policies will take time to transform data culture and improve data sharing capabilities at the national level. Since the direction towards greater openness is already underway, improving the capacity of health practitioners and researchers to use these new data sources effectively is a critical step that needs to be addressed.

How data are interpreted will vary according to the knowledge and experience of the individual user and the way the information is presented. Maps of likely hotspots or regions at risk provide a simple visual tool for decision-makers. However, all maps simplify reality and, because of this, learning to read such maps and understand the information that has been emphasized or neglected in their creation is important in order to make valid inferences. Trust is at the heart of information uptake. Ensuring that sources of data are authoritative and provided with associated meta-data (a set of data that describes and gives information about the data being considered) is key. A healthy scepticism is a valuable asset when exploring new and unfamiliar data sources. 


\subsection{Exploring relationships}

The impact of climate and weather on health is often not immediate. Even when deaths occur from drownings associated with unusually heavy rains there will likely be a delay of hours to days between rain falling and floods occurring - as water takes time to move down rivers and tributaries.

For a vector-borne disease such as Zika, the population dynamics of the vectors (Aedes aegypti and Ae. Albopictus) and virus need to be taken into account when exploring lags between climate drivers and health indicators. In addition to delays associated with vector and pathogen dynamics, the development of a seasonal or epidemic wave is largely attributable to the changing proportion of susceptible hosts in the population (see Figure 3.2). Further, lags in the relationship of climate and health outcomes may be attributed to delays in the manifestation of the disease - e.g., if the disease impacts on the foetus in utero and the child is only included as a case after birth. Manifestations of symptoms associated with Zika virus infection in Brazil, including acute rash, Guillain-Barré syndrome and suspected microcephaly, peaked during epidemiological week 17, 26 and 48 respectively. ${ }^{6}$ It is these transmission lags that allow the creation of EWS based on current and historical environmental and climatic data. For vector-borne diseases in locations subject to distinct rainy seasons, the lag between peak rainfall season and peak cases of disease is commonly around two to three months although the duration of the lag will depend on the climatic conditions ${ }^{7}$ including the distribution and intensity of rainfall, as well as temperature and humidity.

The relationship between temperature and disease transmission is even more complex. ${ }^{8}$ The impact of temperature on the development rates of organisms is amenable to laboratory as well as observational studies. The basic biological response follows a thermal response curve, i.e., has a lower bound minimum, an optimal temperature and a higher bound maximum. This curve may be estimated for a number of different physiological processes occurring in the pathogen, the

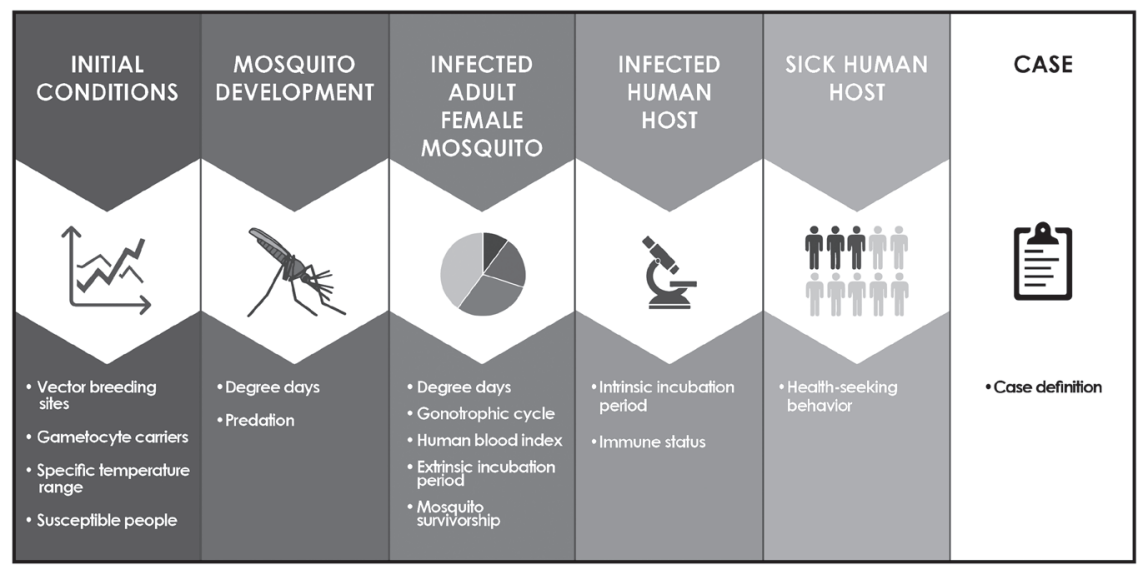

FIGURE 3.2 Understanding lags between climatic events and cases of disease 
vector or the human host and can be compared with field observations. ${ }^{9}$ Not all vector-borne diseases favour warmer climates. Transmission of bubonic plague occurs in cooler mountainous regions (see Case Study 3.1).

\section{CASE STUDY 3.1 PLAGUE, RETURN OF AN OLD FOE Mary Hayden, National Center for Atmospheric Research, Boulder, USA}

Plague (Yersinia pestis) is a bacterial disease that has caused pandemics that have literally changed the course of history; the infamous Black Death, which occurred in the mid-1300s and wiped out a third of Europe's population is one example. Rats and the fleas that they carry have long been viewed as the main sources of human infection resulting in bubonic plague. Human cases have re-emerged in recent years as a result of changing environments and weak or non-existent surveillance. ${ }^{10}$ Human disease usually occurs in one of two forms; bubonic (typically dependent on transmission by fleas) and pneumonic plague which often occurs when bubonic plague victims are not treated and the infection travels to the lungs. Once in the lungs, the disease is spread from person to person through respiratory droplets. More than $90 \%$ of today's plague cases occur in Africa. Plague is commonly found in cooler highland environments where lower temperatures increase the likelihood of transmission to rat or human hosts by the most common vector, the flea Xenopsylla cheopis. Transmission by fleas to rats or humans occurs when the ingested blood meal in their stomach coagulates at temperatures below $27^{\circ} \mathrm{C}$ following the activation of a coagulase enzyme. $Y$. pestis bacteria, which are ingested with the bloodmeal, are able to multiply in the blood clots which are then regurgitated when the flea next takes a bite - allowing the bacteria to penetrate the bite wound and infect the bitten rat or person. At temperatures above $27^{\circ} \mathrm{C}$ coagulase is not produced, and the blood meal does not coagulate; Y. perstis passes through the flea gut and is not regurgitated into the bite wound. As a result, bubonic plague epidemics are not common in environments where temperatures reach above $27^{\circ} \mathrm{C}$.

The West Nile region in northwestern Uganda is a focal point for human plague, which peaks in boreal autumn after the main rainy season. ${ }^{11,12}$ The United States Centers for Disease Control and Prevention (CDC) partnered with the National Center for Atmospheric Research (NCAR) to address the linkages between climate and human plague risk in this region in order to develop a better understanding of potential control options. Because in-situ meteorological records are sparse, a hybrid dynamical-statistical meteorological downscaling technique was applied to generate a multi-year high spatial resolution climate dataset based on NCAR's Weather Research and Forecasting Model. ${ }^{13}$ The dataset was subsequently employed to develop a spatial risk model for human plague occurrence in the West Nile region above 1300 meters, which is cooler and wetter than surrounding areas. ${ }^{14}$ 
Further complicating the statistical analysis of these relationships, temperature variations are often correlated with those of rainfall (see $\$ 5.3 .5$ ).

Given the complex interactions between temperature and malaria transmission it is hard to specify which temperature sensitive aspect of transmission is most important in establishing this lag. As mentioned above it is these lags between observed climatic variables (see $\$ 4.2$ ) and disease indicators that provide the opportunity for the development of climate-informed EWS. When rainfall or temperature changes are predictable, then additional time can be added to the EWS with the use of weather and climate forecasts (see Chapters 7 and 8).

Although for some health outcomes and contexts there may be a strong, linear relationship between a climate driver and cases, the relationship is often highly non-linear. For example, low levels of rainfall $(<2.5 \mathrm{~mm} /$ day $)$ in Botswana appear to have a near-linear relationship to anomalies in malaria cases but a quadratic relationship is clearly observed when wetter conditions are also included in the analysis (Figure 3.3).

The decline in malaria at higher rainfall levels is often attributed to the washing out of mosquito breeding sites during heavy rains. A statistical model developed using moderate to low rainfall years alone would have performed poorly in outof-sample very wet years. In the Botswana example, the most extreme rainfall was associated with cyclone Eline in 2000, a data point that was not used to generate the model. ${ }^{15}$ While heavy rainfall may destroy vector breeding it may create new sites at the end of the rainy season when flood waters retreat. A modelling approach which takes into account the variations in the seasonality of transmission and disease incidence will better capture on-going processes. ${ }^{16}$

An important source of non-linearity in climate-health interactions is the immune response to infection which may protect survivors from re-infection or

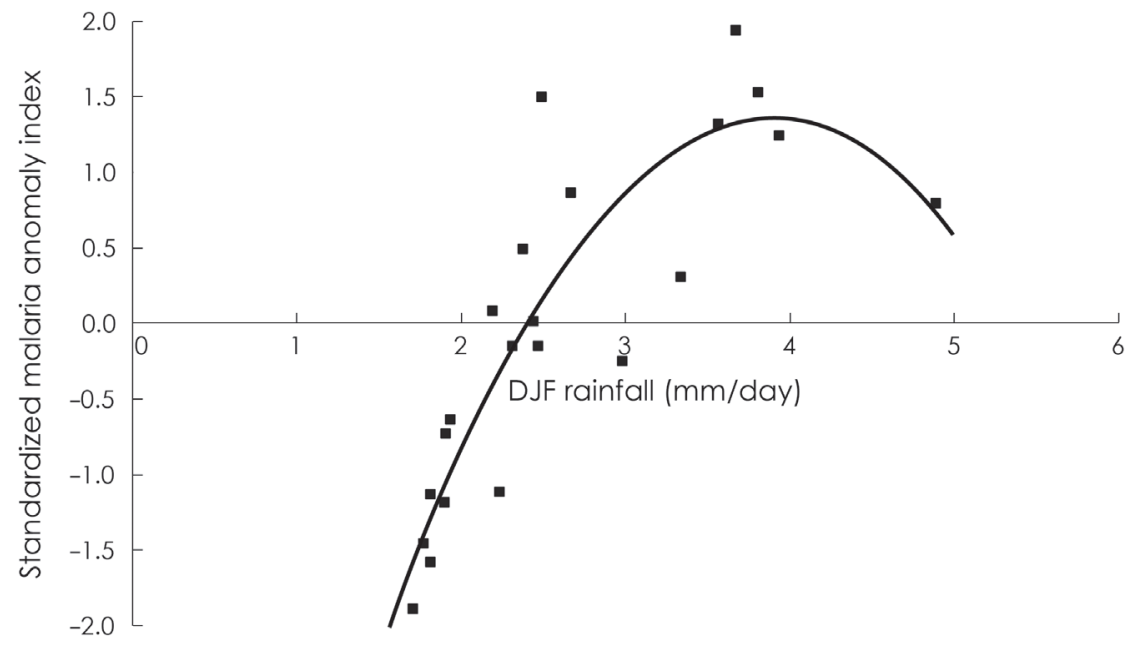

FIGURE 3.3 Relationship between annual malaria anomalies and December to January rainfall in Botswana 
disease for a period of time. The immune status of a population can quickly cause significant changes in the proportion of the population susceptible to infection, and explains why epidemics of infectious diseases often peak and rapidly decline once the source of susceptible individuals is significantly reduced. As higher rates of immunity are commonly found in regions with long or year-round transmission seasons, the impact of climate variability on cases may be effectively buffered. ${ }^{17}$ Statistical models that are based on the assumption that the underlying susceptible population is constant will under-estimate the climate's relationship to disease. Non-linearities in the relationship of climate drivers to disease outcomes are an important reason for considering the use of mathematical models in climate disease analysis as they are able to capture some of these dynamics.

\subsection{Linking climate to health outcomes}

A model attempts to link climate, and possibly other drivers (the model input), to the targeted health outcome (the model output). If the mechanisms and processes that link the two are poorly understood, statistical fitting or 'machine learning' techniques can still be used to provide this link. Alternatively, if a good understanding of the biological processes that drive the health outcome is available, numerical (mechanistic) models can be derived (\$ 8.2.2). As numerical models often make use of statistical approximations when aspects of the model are unknown (just like the parameterizations in weather and climate models; $\$ 7.4 .4$ ), it might be best to consider that these two approaches lie upon a continuum. ${ }^{18}$ The applicability of the approach chosen will depend on characteristics of the pathogen and the hostpathogen relationship as well as the availability of data and information on underlying mechanisms. Of course, it is impossible for the complex systems that we are dealing with (climate and disease) to be exactly represented by any simple model. However, the approximations made by well-chosen models can be extremely useful.

\subsubsection{Statistical models}

Statistical models linking health outcomes and climate exposures, such as climate extremes, varying lengths or intensities of the rainy season, or trends in minimum temperature, can give indications of underlying mechanisms worthy of further exploration, but cannot be used to definitively establish causation because of the problem of confounding variables. Climate may impact, or simply be correlated with, other processes or variables that the researcher is unaware of, and as a consequence the researcher may infer a relationship when there is none. Randomized Controlled Trials (RCTs) are the gold standard study design for the evaluation of medical interventions because they can effectively control for confounding variables. In RCTs participants are randomly allocated between groups to minimize systematic differences between control and interventions groups, and associated biases that might result. In contrast, public health research, including that associated with climate, relies primarily on observational methods. ${ }^{19}$ Because the research is 
often conducted at the population level, it is usually not possible to fully randomize the exposed population on cost, practicality or ethical grounds. As an example, road accidents are positively correlated with monthly temperatures in Europe simply because more and longer journeys are made in the summer months, even though inclement winter weather obviously can cause accidents. In this case, including a denominator in the analysis (number of miles travelled and/or number of drivers on the roads) would reveal the weather-related accident risk to individual drivers.

Systematic reviews from specialist organizations are increasingly used to provide objective and transparent evidence from both RCTs and observational studies (Box 3.2). A systematic approach to reviewing evidence can also inform climate risk, adaptation and mitigation strategies. However unique challenges exist in terms of integrating disparate data as well as analytical norms of different communities. This complexity is illustrated by a systematic review of drought impacts on health. ${ }^{20}$ The review concluded: 'The probability of drought-related health impacts varies widely and largely depends upon drought severity, baseline population vulnerability, existing health and sanitation infrastructure, and available resources with which to mitigate impacts as they occur.'

Climate and health is an emerging field and there may be insufficient peer reviewed literature for a systematic review. Under these circumstances a Delphi review, ${ }^{21}$ where the collective opinion of a group of experts is accessed using a structured process, may be used instead. This approach to critical review is based on the premise that intelligence from a pool of experts can enhance individual judgement if expressed independently. The potential biases associated with observational studies mean that researchers undertaking such studies must pay particular attention to the plausibility of relationships observed. Before using a statistical model to

\section{BOX 3.2 USE OF SYSTEMATIC REVIEWS}

The International Cochrane Collaboration (ICC) ${ }^{i i}$ produces systematic reviews of primary research (usually RCTs) in human health care and health policy and is recognized as the highest standard in evidence-based health care. The ICC investigates the effects of health interventions for prevention, treatment and rehabilitation as well as the effects of diagnostic tests under specific conditions. Other organizations that produce rigorous systematic reviews are: the Campbell Collaboration,iii that produces and disseminates systematic reviews on the effects of interventions in the social and behavioural sciences; the National Institute for Health and Clinical Excellence (NICE), ${ }^{\text {iv }}$ which commissions systematic reviews on new and existing technologies and then uses them to make recommendations to the UK's National Health Service, and the Centre for Reviews and Dissemination (CRD), ${ }^{\vee}$ which produces systematic reviews of health interventions. 
create a forecast, a mechanism by which the observed association might be considered plausible is needed. A number of critical questions that should be asked when developing a decision-support model are proposed in Box 3.3.

\section{BOX 3.3 FIVE QUESTIONS TO CONSIDER WHEN DEVELOP- ING CLIMATE-DRIVEN MODELS FOR DECISION-SUPPORT (ADAPTED FROM ${ }^{22}$ )}

\section{Q1: Do you understand the underlying data?}

Make sure the data used, as well as the processes that generated it, are of the highest possible quality, and that you fully understand them.

\section{Q2: Will the model results will be meaningful to a decision-maker?} Good models usually tell a clear story. If the models you're using don't give you one they may need to be refined. Complex models may be inevitable but they still need to be thought through, refined and simplified enough to make them understandable to those that need to use them.

\section{Q3: Is the model as simple as possible - but not simpler (Occam's Razor)?}

Predictability typically first improves and then deteriorates as model complexity increases, so adding complexity should not be a goal in itself. Of course, there are also risks to oversimplifying the model, so a judgement needs to be made. Einstein is often quoted as saying, 'Everything should be as simple as it can be, but not simpler'; a good principle to apply to predictive analytics.

\section{Q4: Have you tested the predictive accuracy of the model using independent data and across multiple similar environments?}

If the results look to good to be true it's often an over-fitting issue; a common error in predictive analytics. Over-fitting means that the model is too strongly tailored to the data that was used in its development (the training data). Over-fitted models do not predict well new data and therefore cannot make good forecast models. To avoid over-fitting apply the model to fresh data (e.g., through cross validation) in new, but similar, contexts while the model is being developed. A good predictive model should be nearly as accurate with new data as it is with the training set.

\section{Q5: Is the model still relevant?}

Models that have worked well in the past may no longer be relevant (think about economic forecasts!). Because it takes time and energy to develop a model it may be easy to see predictability where there is none. If the data don't support your predictions, you should be prepared to jettison your model - possibly multiple times. Good models are developed through deep understanding of the context for the model development and a very honest interpretation of results. 


\subsubsection{Mathematical models}

Mathematical models of an infectious disease are generally framed around flows between core categories of individuals (e.g., susceptible, exposed, infected, recovered) and sometimes vectors via equations that assume causation and may encompass process uncertainty, climate drivers, etc. An array of options is available to link these mechanistic frameworks to time-series of disease incidence data for a focal infectious disease, e.g., basic maximum likelihood approaches, ${ }^{23}$ Markov Chain Monte Carlo or iterated filtering-type approaches. ${ }^{24}$ These framings can then be used to test hypotheses about important drivers via comparison of how well the model outputs fit the predictand ${ }^{25}$ and can be used to forecast future incidence modulated by a changing climate. Thus, numerical modelling can contribute to understanding the past, and in particular disentangling the relative role of core drivers as well as predicting the future. For infectious disease models, the goal has frequently been to explore different interventions scenarios in order to inform priority-setting for policy-makers. ${ }^{26}$ However, in recent years there is increasing interest in using models for real-time forecasting, ${ }^{27}$ although there remains a significant gap in the operational readiness of the numerous forecasting systems presented in the literature. ${ }^{28}$

\section{CASE STUDY 3.2 SOURCES OF UNCERTAINTY IN MODELLING CLIMATE AND MALARIA}

\section{Adrian M. Tompkins, Abdus Salam International Centre for Theoretical Physics, Trieste, Italy}

The relative importance of transmission model uncertainty, initial condition uncertainty and driving climate uncertainty (Figure 3.4) has been explored using meteorological and malaria data from a highland tea plantation in Kericho, Kenya situated close to the temperature threshold for transmission. ${ }^{29} \mathrm{~A}$ genetic algorithm was used to calibrate each of these three factors within their assessed prior uncertainty in turn to see which allowed the best fit to a time series of approximately 25 years of confirmed malaria cases (the predictand). The spatial representativeness uncertainty for temperature dominated the uncertainty due to model parameter settings. Initial condition uncertainty played a little role after the first two years and is thus important in the EWS context, but negligible for decadal and climate change investigations. Thus, while reducing uncertainty in the model parameters would improve the quality of the simulations, the uncertainty in the temperature data is critical (see Chapter 6). This result is a function of the mean climate of the location itself and model uncertainty would be relatively more important at warmer, lower altitude locations. Uncertainty in model development is then compounded by uncertainty in the way model outputs are used - either for furthering research or supporting decisions (Figure 3.4). 


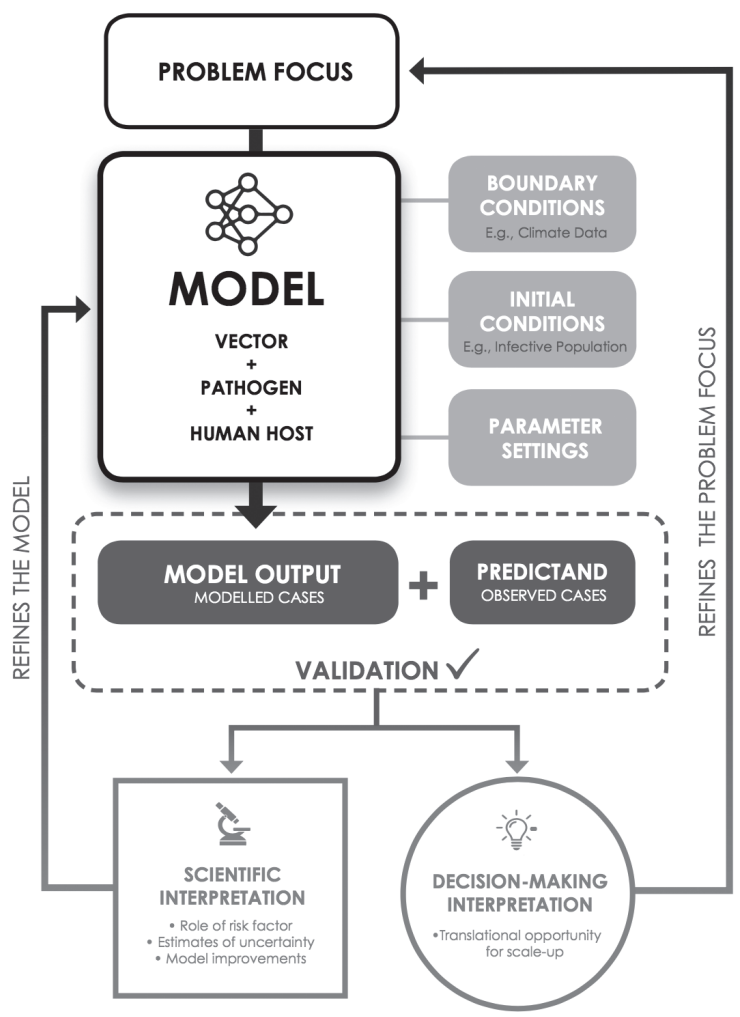

FIGURE 3.4 Schematic of the potential sources of uncertainty when using a weather/ climate-sensitive disease model to simulate observed health outcomes

Because models can only be an approximation of the truth, statisticians and mathematicians have developed modelling tools that can express key elements of the uncertainty in the model. In deterministic models, the output of the model is decided by the parameter values and the initial conditions. However many times you run the model with the same inputs you will get the same result. A stochastic model is a tool used for accounting for known uncertainties in one or more of the parameter values by allowing for random variation over time. The same set of parameter values and initial conditions will lead to an ensemble of different outputs (see Box 7.6). Stochastic models possess some inherent randomness and are used in climate science as well as in disease modelling as they provide an assessment of the range of likely outcomes.

Policy-makers and practitioners need to know how certain the data are that they are using to drive decisions. Understanding sources of uncertainty in the development of climate-driven disease forecasts helps decision-makers understand where, when and why forecasts may be more or less robust and allows the prioritization of error reduction. 
When using a weather-sensitive disease model to simulate observed health outcomes, uncertainty may derive from a variety of sources including the driving climate/environmental data (termed boundary conditions), the entomological and epidemiological initial state (termed initial conditions), the model structure and parameter settings, and lastly errors in the health data itself (the predictand) (see Case Study 3.2).

Aspects of uncertainty are inadequately considered in much epidemiological research ${ }^{30}$ but are usually considered in climate modelling. ${ }^{31} \mathrm{~A}$ common approach taken by the climate community in the development of seasonal climate forecasts is to employ an ensemble of models to create a probability distribution of possible outcomes (see $\$ 7.4$ ). The basic idea is that biases due to imperfect models will tend to cancel each other out. Sometimes probability distributions will be broad and there will be little predictability in the system. However, where there is a sharp probability distribution, predictability is stronger (assuming that the ensemble forecast system is well-calibrated) and the information may be used by decision-makers for taking precautionary action. ${ }^{32}$ The main reason for using a probabilistic system is that users should not be misled by overconfident forecasts. Multi-model ensemble approaches are increasingly being used in the development of health EWS. ${ }^{32-34}$ However, a common challenge to weather/climate and health forecasting is that information on the current situation is required for model initialization ${ }^{35}$ and relevant epidemiological and entomological information is rarely available in near real-time, if at all.

\subsection{Working with uncertain forecasts}

Working with uncertain information is a decision-making problem: the forecaster's job is to try to quantify and minimize the uncertainty in the level of risk and timing of a hazard or event, while it is the practitioner's job to manage that risk and the uncertainty associated with it. Questions of how to manage uncertainties occur in all walks of life, including in public health management: when will the next flu epidemic occur, for example, and how many people will be affected? In Chapter 6, the availability of climate data to estimate public health risk is discussed in detail. Such information is useful for knowing which hazards to worry about at which time of the year, and perhaps how the risks have changed over the last few years. However, ideally we would like to know what the risks are in the coming days (Chapter 7), weeks or season (Chapter 8) or years to decades (Chapter 9), and how they differ from what might be considered normal.

There are some hazards that we have to be prepared for all the time, such as earthquakes, which have no seasonal pattern and can strike without warning. However, it could be exceptionally inefficient if we were having to worry constantly about whether there is likely to be a major storm tomorrow simply because it is the middle of the wet season right now. Forecasts reduce the uncertainty in the risk, making management of that risk easier (but not necessarily easy). Knowing the accuracy or reliability of the forecasts is a prerequisite to identifying the best ways of managing the risk (the distinction between accurate and reliable forecasts is explained in Box 7.4). 
Probabilistic forecasts (see Box 7.5) can maximize the time available to prepare, while minimizing the risk of a false alarm to a level considered acceptable by decision-makers. In the United Kingdom, heat alerts are triggered when there is a $60 \%$ probability of critical day- and night-time temperature thresholds being reached on at least two consecutive days. UK forecasts usually reach the minimum 60\% confidence level two to three days before a heat wave hits, but when a confident forecast is achieved with a longer lead-time, an alert could be given earlier. ${ }^{36}$

Assessing the effectiveness of a climate-informed intervention is more problematic than assessing the direct impact of the climate event alone. At its most basic, evidence of the utility of a EWS requires that morbidity and mortality from a predicted event are compared with a realistic assessment of the hypothetical outcome if the early warning intervention had not been in place. Put another way, one must be able to discern between EWS 'false alarms' and non-occurring 'epidemics' that were prevented by timely action based on the system. A comparison between what actually happened and what would have happened in the absence of the intervention is known as a counter-factual analysis. The simplest approach is to compare the impact of a prior event with a EWS, on the one hand, and an event without a EWS on the other (sometimes referred to as using 'analogues'). However, such comparisons are methodologically problematic because two climatic/weather events are never identical and many other changes to community vulnerability may have happened during the intervening period that may account, at least in part, for the changes in health outcomes. Climate-driven models which can be used to predict what would have happened in the absence of the intervention are best placed to create the counter factual for an EWS. ${ }^{37}$

\subsection{Conclusions}

This chapter highlights the need to understand the spatial-temporal scales of both the decision-context and the potentially relevant climate information. Attention has also been given to specific challenges that are associated with data issues and the identification of climate-health relationships. This and subsequent chapters highlight the need to understand the drivers of uncertainty in model development, since this understanding provides the basis for reducing it where possible. Translating research into policy and practice is a critical consideration for those engaged in developing climate services for the health sector (see $\$ 10.3$ ). The importance of employing a systematic approach to building an evidence-base that can influence policy cannot be over-emphasized.

\section{Notes}

i https://www.cdc.gov/mmwr/preview/mmwrhtml/mm5828a3.htm.

ii www.cochrane.org.

iii https://www.campbellcollaboration.org.

iv https://www.nice.org.uk.

v https://www.york.ac.uk/crd/. 


\section{References}

1 Thomson, M. C., Ceccato, P., Lyon, B. \& Macfarlane, S. B. in The Palgrave Handbook of Global Health Data Methods for Policy and Planning (eds. S. B. Macfarlane \& C. Abouzahr) (Palgrave Macmillan, London, 2018).

2 Nissan, H., Ukawuba, I. \& Thomsom, M. C. Factoring Climate Chamge into Malaria Eradication Strategy. 63pp (GMP WHO, Geneva, 2017).

3 Taylor-Robinson, D. C., Milton, B., Lloyd-Williams, P., O’Flaherty, M. \& Capewell, S. Planning ahead in public health? A qualitative study of the time horizons used in public health decision-making. BMC Public Health 8, 415, doi:10.1186/1471-2458-8-415 (2008).

4 Van Panhuis, W. et al. A systematic review of barriers to data sharing in public health. BMC Public Health 14, 1144, doi:10.1186/1471-2458-14-1144 (2014).

5 Data Revolution Group, UN. A World That Counts: Mobilising the Data Revolution for Sustainable Development. 28pp (2014).

6 Paploski, I. A. D. et al. Time lags between exanthematous Illness attributed to Zika virus, Guillain-Barré Syndrome, and microcephaly, Salvador, Brazil. Emerging Infectious Diseases 22,1438-1444, doi:10.3201/eid2208.160496 (2016).

7 Zhao, X., Chen, F., Feng, Z., Li , X. \& Zhou, X. H. The temporal lagged association between meteorological factors and malaria in 30 counties in south-west China: a multilevel distributed lag non-linear analysis. Malaria Journal 13, 57, doi:10.1186/1475-287513-57. (2014).

8 Paaijmans, K. P., Blanford, S., Chan, B. H. K. \& Thomas, M. Warmer temperatures reduce the vectorial capacity of malaria mosquitoes. Biological Letters 8, 465-468, doi:10.1098/ rsbl.2011.1075 (2012).

9 Mordecai, E. A. et al. Optimal temperature for malaria transmission is dramatically lower than previously predicted. Ecological Letters 16, 22-30, doi:10.1111/ele.12015 (2013).

10 Duplantier, J. M., Duchemin, J. B., Chanteau, S. \& Carniel, E. From the recent lessons of the Malagasy foci towards a global understanding of the factors involved in plague reemergence. Veterinary Research 36, 437-453, doi:10.1051/vetres:2005007 (2005).

11 Eisen, R. J., Griffith, K. S., Borchert, J. N., MacMillan, K., Apangu, T., Owor, N. et al. Assessing human risk of exposure to plague bacteria in northwestern Uganda based on remotely sensed predictors. American Journal of Tropical Medicine and Hygiene 82, 904-911 (2010).

12 Eisen, R. J., Borchert, J., Mpanga, J., Atiku, L., MacMillan, K., Boegler, K. et al. Flea diversity as an element for persistence of plague bacteria in an east African plague focus. PLOS One 7, e35598 (2012) doi: 10.1371/journal.pone.0035598.

13 Monaghan, A. J. et al. A regional climatography of West Nile, Uganda, to support human plague modeling. Journal of Applied Meteorology and Climatology 51, 1201-1221 (2012).

14 MacMillan, K. et al. Climate predictors of the spatial distribution of human plague cases in the West Nile region of Uganda. American Journal of Tropical Medicine and Hygeine 85, 514-523 (2012).

15 Thomson, M. C., Mason, S. J., Phindela, T. \& Connor, S. J. Use of rainfall and sea surface temperature monitoring for malaria early warning in Botswana. American Journal of Tropical Medicine and Hygiene 73, 214-221 (2005).

16 Sofianopoulou, E., Pless-Mulloli, T., Rushton, S. \& Diggle, P. Modeling seasonal and spatiotemporal variation: the example of respiratory prescribing. American Journal of Epidemiology 186, 101-108, doi:10.1093/aje/kww246 (2017).

17 Laneri, K. et al. Dynamical malaria models reveal how immunity buffers effect of climate variability. Proceedings of the Nattional Academy of Sciences 112, 8786-8791, doi:10.1073/ pnas.1419047112 (2015).

18 Metcalf, C. J. E. et al. The seasonal and climatic determinants of access to care: implications for measles outbreak risk in Madagascar. Lancet 389 (2017).

19 Hess, J. J., Eidson, M., Tlumak, J. E., Raab, K. K. \& Luber, G. An evidence-based public health approach to climate change adaptation. Environmental Health Perspectives 122, 1177-1186, doi:10.1289/ehp.1307396 (2014). 
20 Stanke, C., Kerac, M., Prudhomme, C., Medlock, J. \& Murray,V. Health effects of drought: a systematic review of the evidence. PLOS Currents Disasters 5, pii, doi:10.1371/currents. dis.7a2cee9e980f91ad7697b570bcc4b004 (2013).

21 Okoli, C. \& Pawlowski, S. D. The Delphi method as a research tool: an example, design considerations and applications. I. Information Management 42,15-29, doi:10.1016/j. im.2003.11.002. (2004).

22 Evgeniou, T. How to tell if you should trust your statistical models. Harvard Business Review: Information \& Technology (2014). Accessed at https://hbr.org/2014/09/ how-to-tell-if-you-should-trust-your-statistical-models.

23 Bjørnstad, O., Finkenstadt, N. B. \& Grenfell, B. T. Endemic and epidemic dynamics of measles: Estimating epidemiological scaling with a time series SIR model. Ecological Monographs 72, 169-184 (2002).

24 He, D., Ionides, E. L. \& King, A. A. Plug-and-play inference for disease dynamics: measles in large and small populations as a case study. Journal of the Royal Society Interface 7, 271-283 (2010).

25 Laneri, K. et al. Forcing versus feedback: epidemic malaria and monsoon rains in northwest India. PLOS Computational Biology 6, e1000898 (2010).

26 Heesterbeek, H. et al. Modeling infectious disease dynamics in the complex landscape of global health. Science 347, aaa4339. (2015).

27 Yang, W., Karspeck, A. \& Shaman, J. Comparison of filtering methods for the modeling and retrospective forecasting of influenza epidemics. PLOS Computational Biology 10, e1003583. (2014).

28 Corley, C. D. et al. Disease prediction models and operational readiness. PloS One 9, e91989, doi:10.1371/journal.pone.0091989 (2014).

29 Omumbo, J., Lyon, B., Waweru, S. M., Connor, S. \& Thomson, M. C. Raised temperatures over the Kericho tea estates: revisiting the climate in the East African highlands malaria debate. Malaria Journal 10, 12, doi:10.1186/1475-2875-10-12 (2011).

30 Araujo Navas, A. L., Hamm, N. A. S., Soares Magalhães, R. J. \& Stein, A. Mapping soil transmitted helminths and schistosomiasis under uncertainty: a systematic review and critical appraisal of evidence. PLoS Neglected Tropical Diseases 10, e0005208, doi:10.1371/ journal.pntd.0005208 (2016).

31 Palmer,T. N., Doblas-Reyes, F. J., Hagedorn, R. \& Weisheimer, A. Probabilistic prediction of climate using multi-model ensembles: from basics to applications. Philosophical Transactions of the Royal Society B-Biological Sciences 360, 1991-1998 (2005).

32 Thomson, M. C. et al. Malaria early warnings based on seasonal climate forecasts from multi-model ensembles. Nature 439, 576-579 (2006).

33 Ruiz D. et al. Multi-model ensemble (MME-2012) simulation experiments: exploring the role of long-term changes in climatic conditions in the increasing incidence of Plasmodium falciparum malaria in the highlands of Western Kenya. Malaria Journal 13, 206, doi:10.1186/1475-2875-13-206 (2014).

34 Yamana, T. K., Kandula, S. \& Shaman, J. Superensemble forecasts of dengue outbreaks. Journal of the Royal Society Interface 13, pii: 20160410, doi:10.1098/rsif.2016.0410 (2016).

35 Mason, S. J. in Climate Information for Public Health Action (eds. M.C. Thomson \& S. J. Mason), Ch. 7 (Routledge, London, 2018).

36 PHE. Heatwave plan for England. Report No. Accessed at https://www.gov.uk/government/uploads/system/uploads/attachment_data/file/429384/Heatwave_Main_ Plan_2015.pdf, 45 (Public Health England, 2015).

37 Worrall, E., Connor, S. \& Thomson, M. C. A model to simulate the impact of timing, coverage and transmission intensity on effectiveness of indoor residual spraying (IRS). Tropical Medicine and International Health 12, 1-14 (2007). 